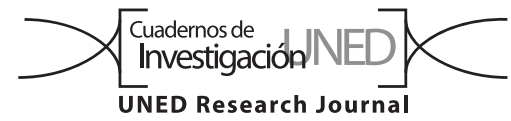

\title{
Composición y riqueza de avifauna en cuatro tipos de cobertura vegetal de San Ramón, Costa Rica
}

\author{
Osvaldo Araya Céspedes ${ }^{1} \&$ José Pablo Carvajal Sánchez ${ }^{1,2}$ \\ 1. Universidad Técnica Nacional, Atenas, Costa Rica; woarayac@est.utn.ac.cr \\ 2. Universidad Nacional, Instituto Internacional en Conservación y Manejo de Vida Silvestre, Heredia; jcarvajals@utn.ac.cr
}

Recibido 04-X-2018 • Corregido 27-I-2019 • Aceptado 30-I-2019

\begin{abstract}
Avifauna composition and richness in four vegetation types, San Ramón, Costa Rica". Introduction: wild birds are important for humans as biological control and seed dispersers, among others. In turn, they can be affected by changes in landscape structure. Objective: to analyze the composition and richness of avifauna in four vegetation types of San Ramón, Costa Rica, between May and August 2016. Methods: point sampling with a fixed $25 \mathrm{~m}$ radius $(75 \mathrm{~m}$ between points, $10 \mathrm{~min}$ per observation) produced 548 individuals from 27 families and 75 species. Results: the most abundant species were Ramphocelus passerinii, Turdus grayi, Crotophaga sulcirostris and Troglodytes aedon. Species richness and abundance were higher in pasture with high canopy cover, and in riverine forest, than in live fences and pastures with low canopy cover. Habitats with the most similarity were riverine forest and live fences. Additionally, $39 \%$ of the represented species were insectivorous, $23 \%$ frugivorous, $17 \%$ omnivorous, $16 \%$ carnivorous, $4 \%$ seed eaters, and $1 \%$ was nectar eater. Lastly, five species were "Forest Dependent" and six were "Indicative of Forest". Conclusion: finally, it should be noted that the areas with the highest vegetation coverage, food availability and refuge areas were the ones with the highest number of species and the most similar sites are determined by the connectivity between them.
\end{abstract}

Keywords: habitats, riparian forest, live fences, abundance, richness.
RESUMEN: Introducción: las aves silvestres son de mucha importancia para el ser humano como controladores biológicos, dispersores de semilla entre otros. Estas a su vez se pueden ver afectadas por los cambios en la estructura del paisaje. Objetivo: analizar la composición y riqueza de avifauna en cuatro tipos de cobertura vegetal en una comunidad del distrito de Peñas Blancas, San Ramón, Costa Rica entre mayo y agosto del 2016. Métodos: el muestreo de aves fue realizado mediante puntos de conteo con radio fijo de $25 \mathrm{~m}$ y una distancia entre puntos de 75 m con 10 minutos de observación por punto. Resultados: registramos 548 individuos pertenecientes a 27 familias y 75 especies. Las 4 especies más abundantes fueron: Ramphocelus passerinii, Turdus grayi, Crotophaga sulcirostris y Troglodytes aedon. La riqueza y abundancia puntual fue mayor en pasto natural de alta cobertura vegetal y en bosque ribereño que en cercas vivas y pastos de baja cobertura vegetal. Los hábitats que tuvieron mayor similitud fueron bosque ribereño y cercas vivas, además el $39 \%$ de las especies estuvo representada por el gremio insectívoro, seguido por frugívoro con $23 \%$, omnívoro con $17 \%$, carnívoro con $16 \%$, granívoro con $4 \%$ y nectarívoro con $1 \%$ y cinco de las especies encontradas son dependientes de bosque y seis de ellas indicadoras. Conclusión: finalmente, cabe destacar que las áreas con mayor cobertura vegetal, disponibilidad de alimento y zonas de refugio fueron las que tuvieron mayor cantidad de especies y los sitios de mayor similitud están determinados por la conectividad entre los mismos.

Palabras Clave: hábitats, bosque ribereño, cercas vivas, abundancia, riqueza.
De acuerdo con MARENA (1999), en Costa Rica y otros países de América Central, la actividad agropecuaria es la principal causa de deforestación. Para el año 2014, el área total con uso agropecuario fue de 2406 418,4 hectáreas, lo que representa el $47,1 \%$ del territorio nacional (INEC, 2015).

Conjuntamente, la conversión de los bosques a la agricultura en Mesoamérica ha transformado los paisajes boscosos en paisajes fragmentados y dominados por pasturas y tierras agrícolas (Harvey, Alpízar, Chacón, \&
Madrigal, 2005). Asimismo, dichos cambios pueden tener un impacto negativo en las comunidades de aves, al reducir hábitats naturales, aislar poblaciones de aves, disminuir el tamaño de las poblaciones y aumentar la depredación en los fragmentos de bosque remanentes (Stiles, 1985). Además, estos cambios pueden provocar la extinción local o regional de especies que dependen de bosques continuos y resultar en cambios en la composición de las especies, a través de la colonización de especies oportunistas (Kattan, 2002). 
No obstante, estudios recientes han demostrado que ciertos usos de la tierra tienen el potencial para proporcionar hábitat y otros recursos para una variedad de plantas y animales, lo que podría contribuir significativamente a los esfuerzos de conservación de las mismas en paisajes fragmentados (Estrada, Coates, \& Meritt, 1997). Conjuntamente, Haber (1999) señala que los árboles en los potreros cumplen un papel importante en la conservación de especies de aves silvestres en paisajes fragmentados, al proporcionar refugio, sitios de descanso, anidación y alimento.

Además, las cercas vivas, cortinas rompe vientos y fragmentos de bosque también pueden servir como corredores biológicos para algunas especies en paisajes agropecuarios, mejorando la conectividad del paisaje y ayudando al desplazamiento de las aves silvestres entre parches de bosques naturales remanentes (Areskoug, 2001). Sin embargo cada localidad es muy independiente y puede tener diferentes comportamientos por lo cual se debe estudiar independientemente.

La presente investigación tuvo como objetivo determinar la composición y riqueza de avifauna en cuatro tipos de cobertura vegetal (bosque ribereño, pastura natural con alta densidad de árboles, pastura natural con baja densidad de árboles y cercas vivas,) en la comunidad del Invu, distrito de Peñas Blancas, San Ramón, Costa Rica. Y como objetivos específicos: estimar la diversidad alfa de especies para cada tipo de cobertura vegetal, analizar la tasa de recambio de especies en los cuatro tipos de cobertura y describir el grado de dependencia de bosque, especies indicadoras y los gremios tróficos de las especies observadas.

\section{MATERIALES Y MÉTODOS}

El estudio se realizó en la comunidad del Invu, distrito Peñas Blancas, San Ramón, Alajuela, Costa Rica. Se encuentra a $116 \mathrm{~km}$ de San José y a altitud promedio de $160 \mathrm{msnm}$. La zona se caracteriza por tener una temperatura promedio anual de $26^{\circ} \mathrm{C}$ y una precipitación media anual de $3500 \mathrm{~mm}$, con una estación lluviosa de mayo a diciembre, y una estación seca de enero a abril. Los fragmentos de bosques naturales remanentes y la vegetación nativa predominante pertenecen a la zona de vida del Bosque Húmedo Tropical (Holdridge, 1987).

Actualmente, el paisaje está caracterizado por la actividad agropecuaria, donde se desarrollan principalmente el cultivo de tubérculos, plátano y ganadería.

En el muestreo de avifauna se estudiaron cuatro tipos de hábitats dominantes en el paisaje: bosque ribereño
(BR), pastura natural de alta cobertura de árboles (16$25 \%$ de cobertura arbórea; PAC) y pastura natural de baja cobertura de árboles (1-15\% de cobertura arbórea; PBC) y cercas vivas (CV). Estos hábitats fueron seleccionados intencionalmente mediante visitas de campo a fincas que contaran con la presencia de los mismos.

En cada finca se eligió un tipo de cobertura vegetal, realizando transeptos de $300 \mathrm{~m}$ con puntos de conteo cada $75 \mathrm{~m}$. Para el caso de Pasto de alta cobertura, debido a al tamaño de las fincas se usó un diseño en distinto ( $L$ y parcela cuadrada, Fig. 1). Las observaciones fueron realizadas en puntos de conteo de $25 \mathrm{~m}$ de radio durante un tiempo de 10 minutos por punto. Cada vez que se llegó a un punto de conteo se dejaron pasar lapsos de $5 \mathrm{~min}$, con el fin que las aves se acostumbrasen a nuestra presencia. Los muestreos se efectuaron entre 6:00-9:00a.m. en el periodo de mayo a agosto del 2016.

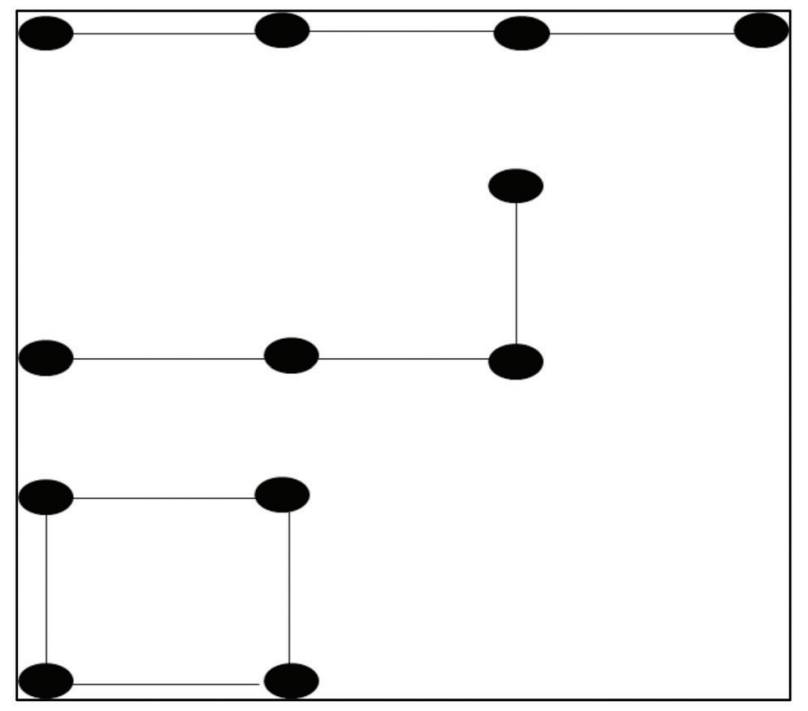

Fig. 1. Diseño de muestreo, (puntos de conteo de aves), en las fincas seleccionadas.

A cada individuo o grupo presente se le registró el número de punto de muestreo, la especie y el número de individuos por especie según Ralph, Geupel et al. (1996). Se calculó la riqueza, abundancia de especies. Además, se realizó un análisis de los hábitats con base en la composición de especies (presencia o ausencia), empleando el índice de similitud de Jaccard; estos índices serán calculados utilizando el software BioDiversity Pro.

Finalmente se clasificaron las aves según el gremio trófico al que pertenecen, distinguiendo entre carnívoras (incluyen carroñeras), frugívoras, granívoras, insectívoras, nectarívoras, omnívoras y piscívoras, de acuerdo con 
la guía de las aves de Costa Rica de Stiles y Skutch (1998). Se determinó el grado de dependencia de bosque de las especies presentes según Stiles (1985) y las especies indicadoras según Dufrene y Legendre (1997).

Ética, conflicto de intereses y declaración de financiamiento: los autores declaran haber cumplido con todos los requisitos éticos y legales pertinentes, tanto durante el estudio como en el manuscrito; que no hay conflictos de interés de ningún tipo, y que todas las fuentes financieras se detallan plena y claramente en la sección de agradecimientos. Asimismo, están de acuerdo con la versión editada final del documento. El respectivo documento legal firmado se encuentra en los archivos de la revista.

\section{RESULTADOS}

Se registraron 548 individuos pertenecientes a $27 \mathrm{fa}$ milias y 75 especies. Las familias con mayor número de especies fueron Tyrannidae con 12 y Thraupidae con 11 respectivamente. Las cuatro especies más abundantes fueron: Ramphocelus passerinii, Turdus grayi, Crotophaga sulcirostris y Troglodytes aedon que se caracterizan por ser especies generalistas.

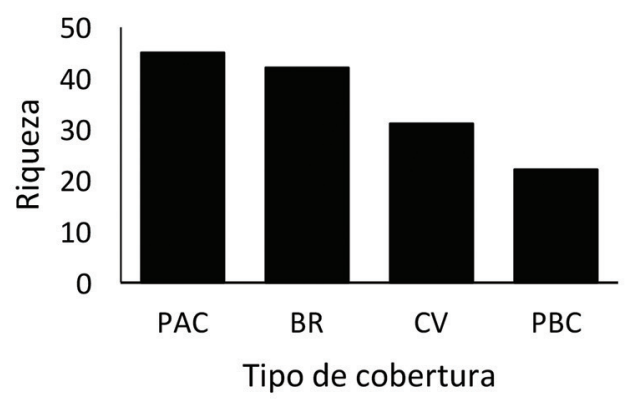

Fig. 2. Riqueza de especies según el tipo de cobertura vegetal.

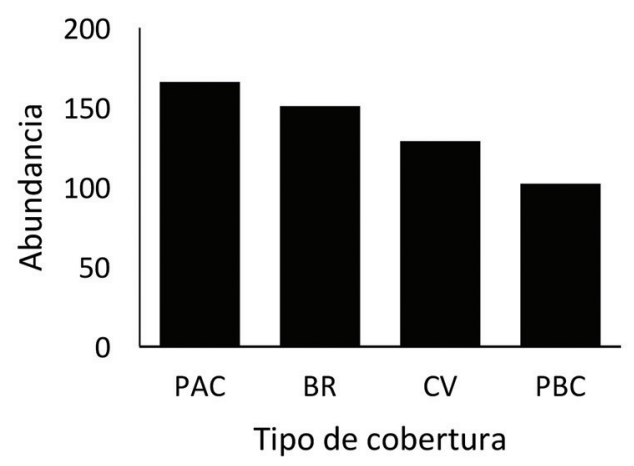

Fig. 3. Abundancia de especies según el tipo de cobertura vegetal para un paisaje de Peñas Blancas, Alajuela, 2016.
Riqueza y abundancia de especies: la riqueza y abundancia puntual fue mayor en pasto natural de alta cobertura vegetal y en bosque ribereño que en cercas vivas y pastos de baja cobertura vegetal.

Beta diversidad: del Cuadro 1 se puede resaltar que los hábitats que tuvieron mayor similitud fueron BR-CV y PBC-CV, mientras los que tuvieron menos están representados por BR-PBC y PAC-PBC. En relación al primero se puede destacar que dichas especies compartidas en su mayoría son especies generalistas y no así las dependientes de bosque o pequeños fragmentos de los mismos. Asimismo en el caso de pastos de baja cobertura, donde se registraron especies de áreas abiertas, las cuales no frecuentan bosques como es el caso de Molothrus aeneus y Quiscalus mexicanus, lo cual hace que la similitud con otros tipos de hábitats sea menor.

\section{CUADRO 1}

Índice de similitud de Jaccard, según el número de especies compartidas con el número total de especies exclusivas para un paisaje de Peñas Blancas, Alajuela, 2016

\begin{tabular}{cc} 
Hábitats & Jaccard \\
BR-PAC & 0,43 \\
BR-PBC & 0,25 \\
BR-CV & 0,61 \\
PAC-PBC & 0,29 \\
PAC-CV & 0,43 \\
PBC-CV & 0,51 \\
\hline
\end{tabular}

Especies indicadoras y dependencia de bosque: se identificaron seis especies de aves indicadoras del total de 75 registradas. En el bosque ribereño dos especies, una especie para pasto de alta cobertura, dos especies para pasto de baja cobertura y una especie para cercas vivas. Estas especies presentaron valores de $p<0,05$, por lo que son consideradas como especies indicadoras para este tipo de cobertura vegetal, según lo plantea el índice de indicador de especies (Cuadro 2).

Según el criterio de dependencia de bosque, se encontraron cinco especies del total de 75 especies registradas, como dependientes de bosque. De estas, la única especie con un valor de uno fue Chondrohierax uncinatus, que necesita de bosque casi continuo para subsistir y que este interconectado en un $50 \%$ con otros fragmentos de bosque. En cuanto a las otras 4 especies, están presentan un valor igual a uno y dos, por lo que necesitan de bosque casi sólido y de pequeños fragmentos de bosque para su supervivencia en el paisaje (Apéndice 1). 
CUADRO 2

Especies de aves indicadoras y dependientes de bosque en un paisaje de Peñas Blancas, Alajuela, 2016

\begin{tabular}{lcc}
\multicolumn{1}{c}{ Especie } & $\mathrm{a}$ & $\mathrm{b}$ \\
Ardea alba & 3 & $\mathrm{BR}$ \\
Manacus candei & 2 & $\mathrm{BR}$ \\
Capsiempis flaveola & 3 & $\mathrm{PAC}$ \\
Crotophaga sulcirostris & 3 & $\mathrm{PBC}$ \\
Quiscalus mexicanus & 3 & $\mathrm{PBC}$ \\
Todirostrum cinereum & 2 y 3 & $\mathrm{CV}$ \\
\hline
\end{tabular}

a. Especies dependientes de bosque según el criterio de Stiles (1985). Clase 1 requieren de un 50\% de cobertura de bosque con gran interconexión entre fragmentos de bosque continuo, especies de la Clase 2 pueden persistir en superficies con menos de un 50\% de cobertura de bosque, Clase 3 no requieren de bosque y pueden persistir en paisajes agrícolas.

b. Especies indicadoras según el criterio de Dufrene y Legendre (1997). Con valores de $p<0,05$. Abreviaturas: $B R$, Bosque ribereño; PAC, Pasto alta cobertura; PBC, Pasto baja cobertura; CV, Cercas vivas.

De estas, cuatro fueron registradas únicamente en bosque ribereño (C. uncinatus, Celeus loricatus, Piculus simplex y Henicorhina leucosticta), mientras una fue observada en pasto natural de alta cobertura arborea (Pheugopedius atrogularis). Cabe señalar la importancia que juegan este tipo de hábitats en el paisaje, a pesar de que la cobertura arborea es mayor en bosque ribereño, el grado de similitud que tiene pasto natural de alta cobertura, le permite a algunas especies desplazarse ocasionalmente a dichos sitios en busca de alimento y refugio.

Gremios tróficos: el 39\% de las especies estuvo representada por el gremio insectívoro, seguido por frugívoro con $23 \%$, omnívoro con $17 \%$, carnívoro con $16 \%$, granívoro con $4 \%$ y nectarívoro con $1 \%$.

\section{DISCUSIÓN}

La composición y riqueza de aves tuvo diferencias significativas según el tipo de cobertura vegetal. Sin embargo los pastos de alta cobertura vegetal y bosques ribereños presentaron una mayor riqueza de aves que pasto natural de alta cobertura y cercas vivas. Esto podría deberse a que los primeros presentan mayor cobertura arborea, mayor disponibilidad de alimento y zonas de refugio.

Con respecto a la composición de especies, estudios similares revelan patrones semejantes a los obtenidos en este trabajo, donde las áreas con alta cobertura vegetal contienen mayor riqueza en cuanto a especies de aves, siendo de vital importancia para la conservación de las mismas (Carvajal, 2008; Estrada et al., 1997; Schulze et al., 2004; Waltert, Mardiastuti, \& Muhlenberg, 2004; Cárdenas, Harvey, Ibrahim, \& Finegan, 2003; Vilchez, Harvey, Sánchez, Medina, \& Hernández, 2004). En cuanto a familias más abundantes (Carvajal, 2008; Pérez et al., 2006; Styles \& Skutch, 1998) coinciden con Tyrannidae como la más representativa en el hemisferio occidental.

Por otra parte, el alto porcentaje de similitud entre bosque ribereño y cercas vivas, podría estar ligado con el rol que estas juegan en la conectividad de diferentes hábitats en el paisaje. Además, de las 75 especies de aves, solo 10 (Leptotila verreauxi, Tangara larvata, Thraupis episcopus, Troglodytes aedon, Turdus grayi, entre otras) estuvieron presentes en todos los hábitats; estas especies son de áreas abiertas y generalistas excepto Pteroglossus torquatus que requiere de pequeños fragmentos de bosque. Además, 15 especies de aves fueron registradas una vez en todo el muestreo, sobre todo especies de aves típicas del bosque como (Celeus loricatus, Chondrohierax uncinatus), las cuales fueron encontradas solamente en bosque ribereño.

Conjuntamente se registró Gampsonyx swainsonii, especie que según del Hoyo, Elliott y Sargatal, (1994) y Elizondo (2013) solo se encuentra en Nicaragua y desde Colombia hasta el Río Amazonas, además la misma podría encontrarse en el noroeste de Costa Rica, sin embargo para este caso se encuentra en un área distinta.

En cuanto a los gremios tróficos, en un estudio realizado en la región Huetar Norte de nuestro país, Sánchez (2009) coincide en este estudio y cita al grupo de insectívoros como el más representativo.

Finalmente cabe destacar que debido a la poca cantidad de réplicas y al corto periodo del estudio provocó que se registraran solo la mitad de las especies que han sido observadas en la zona, en días y sitios distintos al periodo de este estudio.

\section{AGRADECIMIENTOS}

Los autores agradecen a las familias Araya y Ugalde por permitirnos realizar el estudio dentro de sus propiedades. Asimismo, a Celia Harvey por facilitarnos material académico y a todos nuestros colegas que mediante consejos y demás nos ayudaron con este proyecto de investigación. 


\section{REFERENCIAS}

Areskoug, V. (2001). Utilization of remnant dry-forest corridors by the native fauna in a pastoral landscape in the Paraguayan Chaco. Centrum för Biologisk Mångfald, 3, 25-38.

Carvajal, J.P. (2008). Ensamble de aves, murciélagos y escarabajos estercoleros en diferentes tipos de cobertura vegetal en un agropaisaje del norte de Costa Rica (Tesis de maestría). Universidad Nacional, Heredia, Costa Rica.

Cárdenas, G., Harvey, C., Ibrahim, M., \& Finegan, B. (2003). Diversidad y riqueza de aves en diferentes hábitats en un paisaje fragmentado en Cañas, Costa Rica. Agroforestería en las Américas, 10, 78-85.

del Hoyo, J., Elliott, A., \& Sargatal, J. (1994). Handbook of the Birds of the World. Barcelona, España: Lynx Ediciones.

Dufrene, M., \& Legendre, P. (1997). Species assemblages and indicator species: the need for a flexible asymmetrical approach. Ecological Monographs, 67, 345-366. DOI: $10.2307 / 2963459$

Elizondo, L. (2013). crbio.cr. Recuperado de http://www.crbio.cr:8080/neoportlweb/species/Gampsonyx\%20 swainsonii

Estrada, A., Coates, R., \& Meritt, D. (1997). Anthropogenic landscape changes and avian diversity at Los Tuxtlas, Mexico. Biodiversity and Conservation, 6, 19-43. DOI: 10.1023/A:1018328930981

Haber, W. (1999). Remnant trees and the conservation of biodiversity in Costa Rican pastures. Agroforestry Systems, 44, 37-68.

Harvey, C., Alpízar, F., Chacón, M., \& Madrigal, R. (2005). Assessing Linkages between Agriculture and Biodiversity in Central America: Historical Overview and Future Perspectives. San José, Costa Rica:The Nature Conservancy Mesoamerican \& Caribbean Region, Conservation Science Program.

Holdridge, L. (1987). Ecología basada en zonas de vida. San José, Costa Rica: IICA.

INEC. (2015). VI Censo Nacional Agropecuario. Recuperado de http://www.inec.go.cr/sites/default/files/documentos / agropecuario/publicaciones/reagropeccenagro2014-ti006.pdf

Kattan, G.H. (2002). Fragmentación: patrones y mecanismos de extinción de especies. Cartago, Costa Rica: Libro Universitario Regional.

MARENA. (1999). Sistema de vedas de especies silvestres nicaragüense que regirá en el año 2000 (Resolución ministerial No. 023.99). Managua, Nicaragua: Ministerio del Ambiente y los Recursos Naturales.

Pérez, A.M., Sotelo, M., Ramírez, F., Ramírez, l., López, A., \& Siria, I. (2006). Conservación de la biodiversidad en sistemas silvopastoriles de Matiguás y Rio Blanco (Matagalpa, Nicaragua). Ecosistemas, 3, 125-141.

Ralph, C.J., Geupel, G., Pyle, P., Martin, T., DeSante, D., \& Milá, B. (1996). Manual de métodos de campo para el monitoreo de aves terrestres. Albany, CA: Pacific Southwest Research Station, Forest Service, U.S. Department of Agriculture.

Sánchez, A. (2009). Ensamble y ocurrencia de aves en bosques sometidos a manejo forestal, Región Huetar Norte, Costa Rica (Tesis de maestría). Universidad Nacional, Heredia, Costa Rica.

Schulze, C.H., Waltert, M., Kessler, P.J., Pitopang, R., Shahabuddin., Veddeler, D., ... Tscharntke, I. (2004). Biodiversity Indicador Group of Tropical Land-Use Systems: Comparing Plants, Birds, and Insects. Ecological Applications, 14, 1321-1333. DOI: 10.1890/02-5409

Stiles, F.G. (1985). Conservation of forest birds in Costa Rica: Problems and perspectives. ICBP Technical Publication, 4, 141-168.

Stiles, F.G., \& Skutch, A. (1998). Guía de Aves de Costa Rica. Heredia, Costa Rica: Editorial INBio.

Vílchez, S., Harvey, C., Sánchez, D., Medina, D., \& Hernández, B. (2004). Diversidad de aves en un paisaje fragmentado. Encuentro, 68, 60-75.

Waltert, M., Mardiastuti, A., \& Muhlenberg, M. (2004). Effects of land use on birds species richness in Sulawesi, Indonesia. Conservation Biology, 18, 1339-1346. DOI: 10.1111/j.1523-1739.2004.00127.x 


\section{APÉNDICE 1}

Especies, Gremio, Afinidad (grado de dependencia de bosque) en los cuatro tipos de cobertura vegetal, Peñas Blancas, Alajuela, 2016

\begin{tabular}{|c|c|c|c|c|c|c|}
\hline ESPECIE & $B R(n=2)$ & $\operatorname{PAC}(n=2)$ & $\operatorname{PBC}(n=2)$ & $C V(n=2)$ & G & A \\
\hline \multicolumn{7}{|l|}{ Accipitridae } \\
\hline Buteo plagiatus & $x$ & & $x$ & & C & 2 \\
\hline Chondrohierax uncinatus & $x$ & & & & C & 1 \\
\hline Elanoides forficatus & & $x$ & & & C & 2 \\
\hline Gampsonyx swainsonii & & $x$ & & & C & 3 \\
\hline Rupornis magnirostris & & $x$ & $x$ & $x$ & C & 3 \\
\hline \multicolumn{7}{|l|}{ Alcedinidae } \\
\hline Megaceryle alcyon & $x$ & & & & C & 3 \\
\hline \multicolumn{7}{|l|}{ Ardeidae } \\
\hline Ardea alba & $x$ & & & & 0 & 3, $\left(\mathrm{BR}^{*}\right)$ \\
\hline Bubulcus ibis & & & $x$ & $x$ & 0 & 3 \\
\hline Cochlearius cochlearius & $x$ & & & & 0 & 3 \\
\hline Tigrisoma mexicanum & $x$ & & & & 0 & 3 \\
\hline \multicolumn{7}{|l|}{ Cardinalidae } \\
\hline Cyanocompsa cyanoides & $x$ & & & & 0 & 3 \\
\hline Habia fuscicauda & $x$ & $x$ & & & 0 & 2 \\
\hline \multicolumn{7}{|l|}{ Cathartidae } \\
\hline Coragyps atratus & $x$ & $x$ & $\mathrm{x}$ & & C & 3 \\
\hline \multicolumn{7}{|l|}{ Columbidae } \\
\hline Columbina talpacoti & & $x$ & & & G & 3 \\
\hline Leptotila verreauxi & $x$ & $x$ & $\mathrm{x}$ & $x$ & G & 2 у 3 \\
\hline Patagioenas flavirostris & $x$ & $x$ & & $x$ & G & 3 \\
\hline \multicolumn{7}{|l|}{ Cuculidae } \\
\hline Coccyzus minor & $x$ & & & & $\mathbf{I}$ & 2 y 3 \\
\hline Crotophaga sulcirostris & & $x$ & $x$ & $x$ & $\mathbf{I}$ & $3,\left(\mathrm{PBC}^{*}\right)$ \\
\hline Piaya cayana & $x$ & $x$ & & $x$ & $\mathbf{I}$ & 2 y 3 \\
\hline Tapera naevia & $x$ & $x$ & & $x$ & $\mathbf{I}$ & 3 \\
\hline \multicolumn{7}{|l|}{ Falconidae } \\
\hline Milvago chimachima & & & & $x$ & C & 3 \\
\hline \multicolumn{7}{|l|}{ Fringillidae } \\
\hline Euphonia hirundinacea & $x$ & & & $x$ & $\mathbf{F}$ & 2 у 3 \\
\hline Euphonia luteicapilla & & $x$ & & & $\mathbf{F}$ & 3 у 3 \\
\hline \multicolumn{7}{|l|}{ Furnariidae } \\
\hline Xenops minutus & $x$ & & & & 0 & 2 \\
\hline Synallaxis brachyura & $x$ & & & & 0 & 3 \\
\hline \multicolumn{7}{|l|}{ Galbulidae } \\
\hline Galbula ruficauda & & $\mathrm{x}$ & & & $\mathbf{I}$ & 2 \\
\hline \multicolumn{7}{|l|}{ Icteridae } \\
\hline Agelaius phoeniceus & & & $\mathrm{x}$ & & 0 & 3 \\
\hline Icterus prosthemelas & & $\mathrm{x}$ & & & 0 & 3 \\
\hline Molothrus aeneus & & & $\mathrm{x}$ & $x$ & $\mathbf{I}$ & 3 \\
\hline Quiscalus mexicanus & & & $x$ & & 0 & $3,\left(\mathrm{PBC}^{*}\right)$ \\
\hline \multicolumn{7}{|l|}{ Phalacrocoracidae } \\
\hline Phalacrocorax brasilianus & $x$ & & & & C & 3 \\
\hline \multicolumn{7}{|l|}{ Picidae } \\
\hline Celeus loricatus & $x$ & & & & $\mathbf{I}$ & 1 у 2 \\
\hline Melanerpes hoffmannii & $x$ & $x$ & & $x$ & $\mathbf{I}$ & 2 y 3 \\
\hline
\end{tabular}


APÉNDICE 1 (Continuación)

\begin{tabular}{|c|c|c|c|c|c|c|}
\hline ESPECIE & $B R(n=2)$ & PAC $(n=2)$ & $\operatorname{PBC}(n=2)$ & $C V(n=2)$ & G & A \\
\hline Melanerpes pucherani & $x$ & $x$ & & & $\mathbf{I}$ & 2 \\
\hline Piculus simplex & $x$ & & & & $\mathbf{I}$ & 1 у 2 \\
\hline Picumnus olivaceus & $\mathrm{x}$ & $x$ & & & $\mathbf{I}$ & 2 y 3 \\
\hline \multicolumn{7}{|l|}{ Pipridae } \\
\hline Manacus candei & $x$ & & & & $\mathbf{F}$ & $2,\left(B R^{*}\right)$ \\
\hline \multicolumn{7}{|l|}{ Psittacidae } \\
\hline Brotogeris jugularis & & $x$ & & & $\mathbf{F}$ & 3 \\
\hline \multicolumn{7}{|l|}{ Rhamphastidae } \\
\hline Pteroglossus torquatus & $x$ & $x$ & $x$ & $x$ & $\mathbf{F}$ & 2 \\
\hline \multicolumn{7}{|l|}{ Strigidae } \\
\hline Glaucidium brasilianus & & $\mathrm{x}$ & & & C & 2 y 3 \\
\hline Pulsatrix perspicillata & $x$ & & & & C & 2 \\
\hline \multicolumn{7}{|l|}{ Thamnophilidae } \\
\hline Thamnophilus doliatus & $x$ & $\mathrm{x}$ & & $x$ & $\mathbf{I}$ & 2 y 3 \\
\hline \multicolumn{7}{|l|}{ Thraupidae } \\
\hline Coereba flaveola & $x$ & $\mathrm{x}$ & & & $\mathbf{F}$ & 2 y 3 \\
\hline Cyanerpes cyaneus & $x$ & $\mathrm{x}$ & & $x$ & $\mathbf{F}$ & 2 \\
\hline Oryzoborus funereus & & $x$ & & $x$ & $\mathbf{F}$ & 3 \\
\hline Ramphocelus passerinii & $x$ & $x$ & $x$ & $x$ & $\mathbf{F}$ & 3 \\
\hline Saltator atriceps & & $x$ & & & $\mathbf{F}$ & 3 \\
\hline Saltator maximus & $x$ & $x$ & & $x$ & $\mathbf{F}$ & 3 \\
\hline Sporophila corvina & & $x$ & $x$ & $x$ & $\mathbf{F}$ & 3 \\
\hline Sporophila torqueola & & $x$ & & & $\mathbf{F}$ & 3 \\
\hline Tangara larvata & $x$ & $x$ & $x$ & $x$ & $\mathbf{F}$ & 3 \\
\hline Thraupis episcopus & $x$ & $x$ & $x$ & $x$ & $\mathbf{F}$ & 3 \\
\hline Thraupis palmarum & & $\mathrm{x}$ & & & $\mathbf{F}$ & 3 \\
\hline \multicolumn{7}{|l|}{ Threskiornithidae } \\
\hline Platalea ajaja & $x$ & & & & C & 3 \\
\hline \multicolumn{7}{|l|}{ Tityridae } \\
\hline Pachyramphus cinnamomeus & & & $x$ & $x$ & 0 & 2 y 3 \\
\hline Tityra inquisitor & & $x$ & & & $\mathbf{I}$ & 2 \\
\hline Tityra semifasciata & $\mathrm{x}$ & $x$ & & $x$ & $\mathbf{I}$ & 2 y 3 \\
\hline \multicolumn{7}{|l|}{ Trochilidae } \\
\hline Amazilia tzacatl & $x$ & $x$ & & $x$ & $\mathbf{N}$ & 3 \\
\hline \multicolumn{7}{|l|}{ Troglodytidae } \\
\hline Henicorhina leucosticta & $x$ & & & & I & 1 у 2 \\
\hline Pheugopedius atrogularis & & $x$ & & & $\mathbf{I}$ & 1 у 2 \\
\hline Troglodytes aedon & $x$ & $x$ & $x$ & $x$ & $\mathbf{I}$ & 3 \\
\hline \multicolumn{7}{|l|}{ Trogonidae } \\
\hline Trogon caligatus & $x$ & $x$ & & & $\mathbf{F}$ & 2 y 3 \\
\hline \multicolumn{7}{|l|}{ Turdidae } \\
\hline Turdus grayi & $x$ & $x$ & $x$ & $x$ & $\mathbf{I}$ & 3 \\
\hline \multicolumn{7}{|l|}{ Tyrannidae } \\
\hline Attila spadiceus & $x$ & & & & $\mathbf{I}$ & 2 \\
\hline Capsiempis flaveola & & $x$ & & & $\mathbf{I}$ & $3,\left(\mathrm{PAC}^{*}\right)$ \\
\hline Contopus sp & $\mathrm{x}$ & $\mathrm{x}$ & $x$ & $\mathrm{x}$ & $\mathbf{I}$ & 3 \\
\hline Legatus leucophaius & $x$ & & & & 1 & 3 \\
\hline Megarynchus pitangua & & $x$ & $x$ & & $\mathbf{I}$ & 2 y 3 \\
\hline Miarchus tubercilifer & & & & & $\mathbf{I}$ & 2 y 3 \\
\hline Myiodynastes luteiventris & & $x$ & & & I & 2 y 3 \\
\hline
\end{tabular}


APÉNDICE 1 (Continuación)

\begin{tabular}{|c|c|c|c|c|c|c|}
\hline ESPECIE & $B R(n=2)$ & PAC $(n=2)$ & $\operatorname{PBC}(n=2)$ & $C V(n=2)$ & G & A \\
\hline Myiozetetes granadensis & & & $x$ & $x$ & $\mathbf{I}$ & 2 y 3 \\
\hline Pitangus sulphuratus & $x$ & $x$ & $x$ & $x$ & 0 & 3 \\
\hline Todirostrum cinereum & $\mathrm{x}$ & $\mathrm{x}$ & $\mathrm{x}$ & $\mathrm{x}$ & $\mathbf{I}$ & 2 у $3,\left(C^{*}\right)$ \\
\hline Tolmomyas sulphurescens & $x$ & & & & I & 3 \\
\hline Tyrannus melancholicus & $x$ & $\mathrm{x}$ & $x$ & $x$ & $\mathbf{I}$ & 3 \\
\hline Total individuos & 151 & 166 & 102 & 129 & & \\
\hline Total especies & 42 & 45 & 22 & 31 & & \\
\hline
\end{tabular}

Tipos de cobertura: $\mathrm{BR}=$ Bosque ribereño, $\mathrm{PAC}=$ Pasto alta cobertura, $\mathrm{PBC}=$ Pasto baja cobertura, $\mathrm{CV}=$ Cerca viva.

$\mathrm{X}$ : especie presente en el tipo de cobertura.

(G) Gremio: C=Carnívoro, F=Frugívoro, G=Granívoro, N=Nectarívoro, l=Insectívoro, O=Ovívoro, H=Herbívoro. Clasificados según Styles y Skutch (1989).

(A) Afinidad: Grado de dependencia. 1=Necesita bosque casi sólido, 2=Necesita pequeños fragmentos de bosque, 3=No necesita el bosque. Indicador de especies: en la columna de Afinidad, las especies indicadoras están mostradas por un asterisco, por ejemplo ( $\left.\mathrm{CV}^{*}\right)$, lo que indica que la especie es indicadora de cercas vivas. Clasificados según el grado de significancia donde si $p<0,05$ las especies son indicadoras según el análisis de Indicador de Especies de Dufrene y Legendre (1997)

\section{APÉNDICE 2}

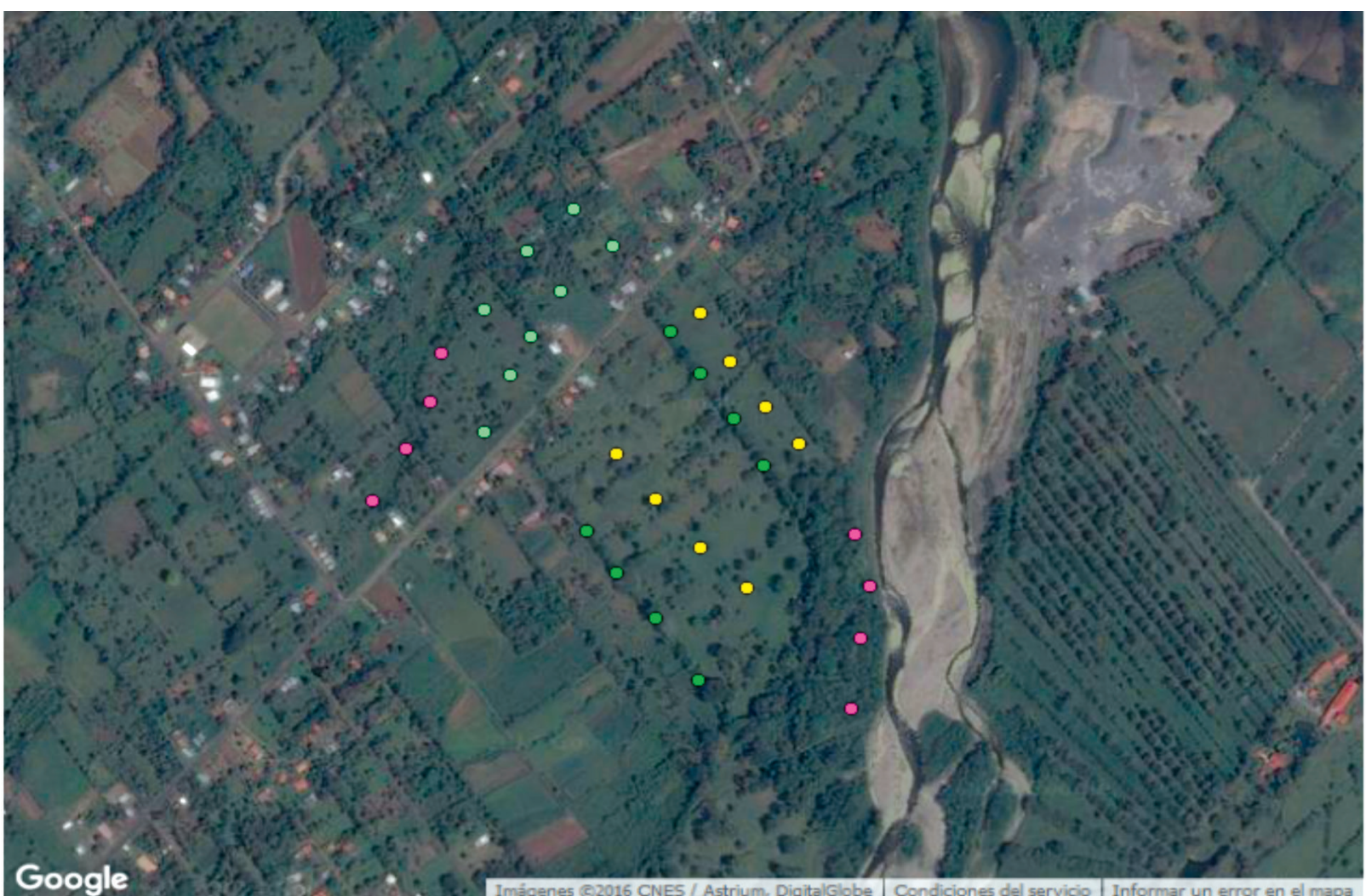

Imagen satelital área de estudio, puntos de conteo (lila $=B R$, verde turquesa $=P A C$, amarillo $=P B C$, verde $=C V$ ) Fuente: Google Earth, 2016. 